\title{
Low accuracy of chromogranin A for diagnosing early-stage pancreatic neuroendocrine tumors
}

\author{
CHAO-MING TSENG ${ }^{1}$, TSU-YAO CHENG $^{2,3}$, TAI-BEEN CHEN $^{4}$, YU-WEN TIEN $^{5}$, \\ CHIEN-CHUAN CHEN ${ }^{3}$, JAW-TOWN LIN ${ }^{6}$ and HSIU-PO WANG ${ }^{3}$
}

${ }^{1}$ Department of Internal Medicine, E-Da Cancer Hospital/I-Shou University, Kaohsiung 824; Departments of ${ }^{2}$ Laboratory Medicine and ${ }^{3}$ Internal Medicine, National Taiwan University Hospital and National Taiwan University College of Medicine, Taipei 10002; ${ }^{4}$ Department of Medical Imaging and Radiological Sciences,

I-Shou University, Kaohsiung 824; ${ }^{5}$ Department of Surgery, National Taiwan University Hospital and National Taiwan University College of Medicine, Taipei 10002; ${ }^{6}$ School of Medicine,

Fu Jen Catholic University, New Taipei City 10002, Taiwan, R.O.C.

Received June 21, 2016; Accepted August 8, 2017

DOI: $10.3892 / \mathrm{ol} .2018 .8472$

\begin{abstract}
The aim of the present study was to evaluate the clinical utility of plasma chromogranin $\mathrm{A}(\mathrm{CgA})$ in patients diagnosed with early-stage pancreatic neuroendocrine tumors (PNETs) in terms of diagnostic value and treatment response. A total of 35 patients with PNETs were prospectively enrolled from August 2010 to April 2014. Demographic and clinicopathological data were collected, and serial plasma CgA levels were measured. Tumor responses were defined by the Response Evaluation Criteria In Solid Tumors criteria. Pearson's $\chi 2$ test was used for the analysis of the association between the plasma $\mathrm{CgA}$ level and various factors. Plasma $\mathrm{CgA}$ level was significantly associated with the size $(\mathrm{P}=0.03)$, metastasis $(\mathrm{P}=0.02)$ and tumor stage $(\mathrm{P}=0.03)$ of the PNETs. Using $126 \mathrm{U} / \mathrm{l}$ as the optimal cutoff value, the sensitivity and specificity were 87.5 and $81.5 \%$, respectively. For localized tumors, the sensitivity of $\mathrm{CgA}$ for diagnosing PNETs was relatively low, even following a lowering of the cutoff values (29.6-51.9\%). Plasma CgA level was correlated with therapeutic response in those patients with
\end{abstract}

Correspondence to: Professor Hsiu-Po Wang or Dr Tsu-Yao Cheng, Department of Internal Medicine, National Taiwan University Hospital and National Taiwan University College of Medicine, 7 Chung-Shan South Road, Taipei 10002, Taiwan, R.O.C. E-mail:wanghp@ntu.edu.tw

E-mail: tycheng@ntu.edu.tw

Abbreviations: CgA, chromogranin A; EUS-FNA, endoscopic ultrasound-guided fine-needle aspiration; NET, neuroendocrine tumor; GEP-NET, gastro-entero-pancreatic NET; MEN-1, multiple endocrine neoplasia type 1; PNET, pancreatic NET; RECIST, Response Evaluation Criteria In Solid Tumors; ROC, receiver operating characteristic; WHO, World Health Organization

Key words: chromogranin A, pancreatic neuroendocrine tumor, tumor marker high baseline $\mathrm{CgA}$ levels ( $\mathrm{P}=0.025)$, but not in the patients with low baseline $\mathrm{CgA}$ levels $(\mathrm{P}=0.587)$. In conclusion, plasma $\mathrm{CgA}$ level was associated with tumor size, metastasis and tumor stage in patients with PNET. For early-stage PNETs, CgA exhibited a limited role in diagnosis and treatment response evaluation in the population of the present study.

\section{Introduction}

Pancreatic neuroendocrine tumors (PNETs) are rare neoplasms, with an incidence of 0.48 cases $/ 100,000$ individuals each year between 2000 and 2012 in the United States of America (1-2). A trend towards increasing incidence and prevalence rates has been documented by previous studies $(3,4)$. Although functional PNETs may be present in a variety of hormone syndromes and detected at an earlier stage, numerous non-functional PNETs are diagnosed late in the disease course, with symptoms associated with local mass effects or metastatic disease (5). A biomarker with a high sensitivity and specificity is mandatory for the early and accurate diagnosis of PNETs, particularly for those with vague symptoms.

Chromogranin A $(\mathrm{CgA})$ is an acidic glycoprotein stored in the dense granules of the NETs and co-released with peptide hormones (6). $\mathrm{CgA}$ has been suggested to be a reliable biomarker for NETs in terms of diagnostic value, prognosis prediction and treatment response evaluation $(7,8)$. In western countries, a low diagnostic accuracy but good prognostic value of $\mathrm{CgA}$ in patients with resectable non-functional PNETs has been demonstrated $(9,10)$. Concerning differences in biomarker performance across racial groups, there are a small number of studies examining the diagnostic value of $\mathrm{CgA}$ in Asian populations. Plasma $\mathrm{CgA}$ has been suggested to be a useful biomarker for PNETs in Asian populations (11-14), but few studies have evaluated the diagnostic role of plasma $\mathrm{CgA}$ in early-stage PNETs. The present study aimed to evaluate the clinical utility of plasma $\mathrm{CgA}$ in the diagnostic confirmation and evaluation of treatment response in Asian patients with PNETs, particularly those with early-stage tumors. 


\section{Materials and methods}

Study design and population. The study protocol was approved by the Institutional Review Board of National Taiwan University Hospital (Taipei, Taiwan). Written informed consent for participation in the study was obtained from all participants. From August 2010 to April 2014, 35 patients with PNETs according to tissue-based diagnosis were prospectively enrolled consecutively. The age, gender, clinical presentation and plasma $\mathrm{CgA}$ level were recorded prior to tissue-based diagnosis Patients presenting with symptoms suggestive of excessive hormone production were considered to have functional PNETs, and the other patients were considered to have non-functional tumors. Endoscopic ultrasound-guided fine-needle aspiration (EUS-FNA) cell blocks or surgical specimens were used for a definite diagnosis. The tissue coagulum clot method was adapted for cell block preparation (15). The steps included fixation by transferring tissue coagulum clot strips into the $10 \%$ formalin solution at room temperature for between 10 and $24 \mathrm{~h}$, subsequent centrifugation, and final transfer of the pellet for paraffin embedding as a cell block. Each cell clock was examined via hematoxylin and eosin staining and immunohistochemical staining with antibodies against $\mathrm{CgA}$ and synaptophysin, as previously described (16). Histopathological characteristics, including tumor location, size, grade, Tumor-Node-Metastasis (TNM) status and tissue $\mathrm{CgA}$ immunoreactivity were also recorded. Tumor grade was classified according to the recommendations of the World Health Organization (WHO) (17). TNM status was based on the 7th edition of the American Joint Commission on Cancer Staging System (18). Inclusion criteria included patients aged $\geq 20$ years old with tissue-based diagnosis of PNETs consecutively within the study period. Participants were recruited on a rolling basis as and when they were identified using tissue-based diagnosis. Exclusion criteria included end-stage renal disease, liver failure and the presence of any other malignancies. Serial $\mathrm{CgA}$ measurement and imaging studies were used to evaluate treatment response. Response Evaluation Criteria In Solid Tumors (RECIST) (19) was used to evaluate the treatment response of the image studies.

Measurement of plasma $\mathrm{CgA}$. Blood samples were obtained following overnight fasting and collected prior to definite tissue-based diagnosis either by EUS-FNA or by surgical resection. The plasma $\mathrm{CgA}$ level was measured with a commercial kit (Chromoa R assay; CIS Bio International S.A., Saclay, France; cat. no. CGA-ELISA), according to the manufacturer's protocol. The recommended cutoff value was set at $94 \mathrm{U} / 1$, according to a previous study (9).

Statistical analysis. Comparison of values from independent groups was performed using a Mann-Whitney test. Pearson's $\chi^{2}$ test was used to measure the strength of the association between pairs of variables without specifying dependencies. To determine whether $\mathrm{CgA}$ level was an independent predictor of metastasis, hazard ratios were calculated using the Cox proportional hazards model. The sensitivity and specificity of $\mathrm{CgA}$ level to discriminate metastatic from localized PNETs were analyzed using the receiver operating characteristic
(ROC) curve, and the optimal cutoff value was determined. Kendall's $\tau$ correlation test was applied to estimate the correlation between change of $\mathrm{CgA}$ level and treatment response by RECIST. Data were analyzed with SPSS version 17.0 (SPSS Inc., Chicago, IL, USA). $\mathrm{P}<0.05$ was considered to indicate a statistically significant difference.

\section{Results}

Clinical characteristics of enrolled patients. Among the 35 patients with PNET, there were 18 males and 17 females. The mean age of these patients was 53.0 (range, 31-80) years. A total of 29 patients received surgical resection only, and 1 patient received surgical resection and additional targeted therapy. A total of 4 patients received systemic antitumor therapies, including target therapy and cytotoxic chemotherapy, and 1 patient received best supportive care. The $\mathrm{CgA}$ levels among patients with PNET with different clinicopathological characteristics were compared (Table I). There was no association between sex and $\mathrm{CgA}$ value. The patients usually presented asymptomatically, and those PNETs were identified incidentally by health examination $(21 / 35,60 \%)$. The majority of these tumors were well differentiated (G1; 22/35, $63 \%)$ and localized tumors (27/35, 77\%). Among patients with the localized disease, the majority of the tumors were $>2 \mathrm{~cm}$ in diameter $(16 / 27,59 \%)$. The majority of the tumors were located in single areas of the pancreatic body or tail where they were more easily detected by routine abdominal sonography, with the exception of 3 cases of multiple endocrine neoplasia type 1 (MEN-1), which presented with multiple tumors from the pancreatic head to tail. The majority of the patients with PNETs were immunoreactive to tissue $\mathrm{CgA}$.

Univariate analysis of $\mathrm{CgA}$ levels based on the clinicopathological variables demonstrated that plasma $\mathrm{CgA}$ levels were significantly higher in patients with larger tumor size (size $>2 \mathrm{~cm}$ vs. size $\leq 2 \mathrm{~cm}$ : 115.4 vs. $64.0 \mathrm{U} / \mathrm{l}, \mathrm{P}=0.03$; Fig. 1A), distant metastasis (metastasis vs. non-metastasis: 308.7 vs. $67.9 \mathrm{U} / 1, \mathrm{P}=0.02$; Fig. $1 \mathrm{~B}$ ) and advanced pathological stage (stage II/III/IV vs. stage I: 221.0 vs. $66.5 \mathrm{U} / 1, \mathrm{P}=0.03$; Fig. 1C). CgA level did not differ in terms of sex, age, clinical symptoms, proton pump inhibitor use, MEN-1 presence, tumor location, tumor grade or tissue $\mathrm{CgA}$ immunoreactivity.

Diagnostic value of $C g A$. Table II summarizes the sensitivities of plasma CgA levels of PNETs under the different cutoff values suggested by previous studies $(8,12,16)$. When the cutoff value was $94 \mathrm{U} / 1$, the overall sensitivity of plasma $\mathrm{CgA}$ was only $42.9 \%$. Subgroup analysis indicated that the sensitivity of plasma $\mathrm{CgA}$ for patients with metastatic tumors was $87.5 \%$, while the sensitivity for patients with localized tumors was $29.6 \%$. On evaluation under different cutoff values, all the sensitivities for the patient with localized PNETs were poor (74 U/1, 40.7\%; $65.7 \mathrm{U} / 1,51.9 \%$ ).

CgA as a predictor of metastasis. Table III summarizes the univariate and multivariate analyses of predictors of metastasis. Larger tumor size ( $\mathrm{P}=0.019)$, WHO grade 2 or 3 $(\mathrm{P}=0.006)$ and higher plasma $\mathrm{CgA}$ level $(\geq 94 \mathrm{U} / 1 ; \mathrm{P}=0.014)$ were associated with a higher risk of metastasis according to the univariate analysis. Multivariate analysis of significant 
Table I. Plasma CgA levels in patients with PNETs.

\begin{tabular}{|c|c|c|c|}
\hline Variables & $\mathrm{n}$ & Median CgA level (range), U/l & P-value \\
\hline Sex & & & 0.22 \\
\hline Female & 17 & $84.4(12.1-16465.7)$ & \\
\hline Male & 18 & $66.5(33.6-3117.5)$ & \\
\hline Age, years & & & 0.26 \\
\hline$<50$ & 17 & $67.9(12.1-16465.7)$ & \\
\hline$\geq 50$ & 18 & $107.5(33.6-3117.5)$ & \\
\hline Clinical symptoms & & & 0.71 \\
\hline Absent & 21 & $53.5(12.1-3117.5)$ & \\
\hline Present & 14 & $70.5(99.5-16465.7)$ & \\
\hline Proton pump inhibitor & & & 0.39 \\
\hline Absent & 33 & $84.4(12.1-3117.5)$ & \\
\hline Present & 2 & $8264.4(63.0-16465.7)$ & \\
\hline MEN-1 & & & 0.68 \\
\hline Absent & 32 & $85.0(12.1-3117.5)$ & \\
\hline Present & 3 & $67.9(59.9-16465.7)$ & \\
\hline Pancreatic location $^{\mathrm{a}}$ & & & 0.62 \\
\hline Body + tail & 21 & $72.7(33.6-3117.5)$ & \\
\hline Head + neck & 11 & $86.5(12.1-2637.5)$ & \\
\hline Size, $\mathrm{cm}$ & & & 0.03 \\
\hline$\leq 2$ & 16 & $64.0(12.1-1506.5)$ & \\
\hline$>2$ & 19 & $115.4(33.6-16465.7)$ & \\
\hline Metastasis & & & 0.02 \\
\hline Absent & 27 & $67.9(12.1-16465.7)$ & \\
\hline Present & 8 & $308.7(35.9-2637.5)$ & \\
\hline Pathological stage & & & 0.03 \\
\hline I & 24 & $66.5(12.1-16465.7)$ & \\
\hline $\mathrm{II}+\mathrm{III}+\mathrm{IV}$ & 11 & $221.0(35.9-2637.5)$ & \\
\hline WHO grade & & & 0.59 \\
\hline G1 & 22 & $72.7(42.5-16465.7)$ & \\
\hline $\mathrm{G} 2+\mathrm{G} 3$ & 13 & $132.0(12.1-3117.5)$ & \\
\hline Tissue CgA & & & 0.20 \\
\hline Negative & 2 & $51.4(35.9-67.9)$ & \\
\hline Positive & 33 & $85.5(12.1-16465.7)$ & \\
\hline
\end{tabular}

${ }^{\mathrm{a}} \mathrm{A}$ total of $3 \mathrm{MEN}-1$ cases were excluded due to multiple locations. PNET, pancreatic neuroendocrine tumor; CgA, chromogranin A; MEN-1, multiple endocrine neoplasia type 1; WHO, World Health Organization.

factors indicated that only higher plasma $\mathrm{CgA}$ level was significantly associated with a higher risk of metastasis $(\mathrm{P}=0.045)$. To distinguish between patients with localized disease or metastasis, a ROC curve analysis was applied (Fig. 2). When the cutoff value was $94 \mathrm{U} / 1$, the sensitivity and specificity were 87.5 and $70.4 \%$, respectively. Using $126 \mathrm{U} / 1$ as the optimal cutoff value, the sensitivity and specificity were 87.5 and $81.5 \%$, respectively.

Correlation between plasma CgA change and treatment response. A total of 29 out of 35 patients underwent serial $\mathrm{CgA}$ measurement and imaging studies prior and subsequent to treatment (6 patients did not receive serial CgA measurement as they were lost to follow-up). A total of 13 patients exhibited high baseline plasma CgA levels $(\geq 94 \mathrm{U} / \mathrm{l})$ and 16 patients exhibited low baseline levels $(<94 \mathrm{U} / \mathrm{l})$. Among the patients with high baseline $\mathrm{CgA}$ levels, 7 patients achieved a complete response and 3 patients demonstrated a partial response. A total of 3 patients exhibited progressive disease. Serial plasma $\mathrm{CgA}$ change was correlated with treatment response. A decrease of $\geq 30 \%$ in CgA levels was observed in patients with complete and partial responses $(\mathrm{P}=0.025$; Fig. $3 \mathrm{~A})$. Among the patients with low baseline $\mathrm{CgA}$ levels, 15 patients achieved complete response and 1 patient exhibited progressive disease. However, serial $\mathrm{CgA}$ level was not correlated with treatment response in this patient group $(\mathrm{P}=0.587$; Fig. $3 \mathrm{~B})$. 


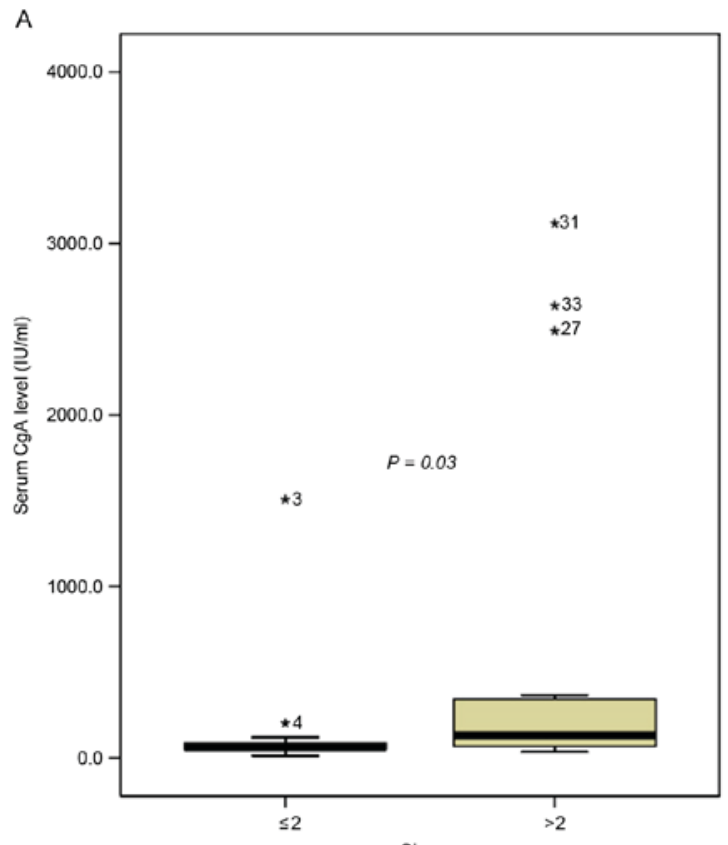

B

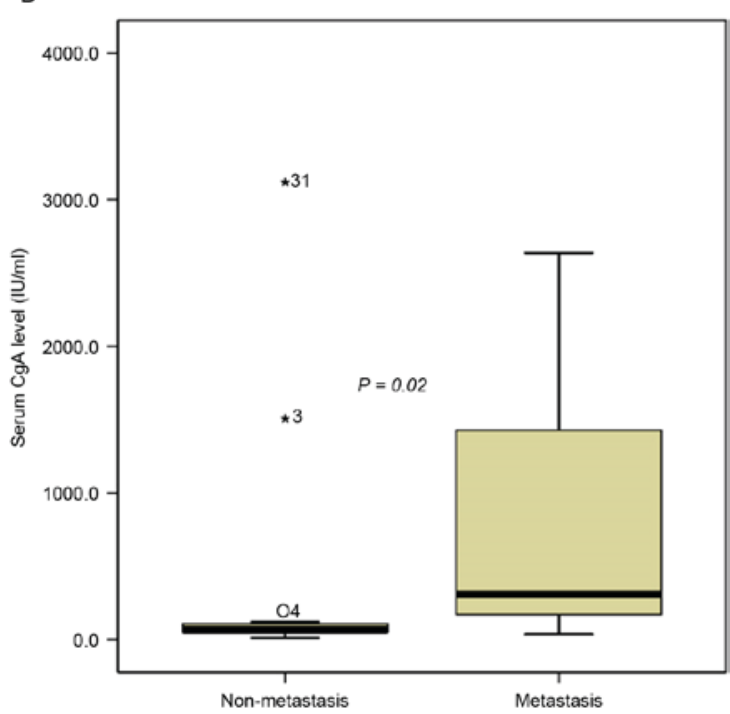

C

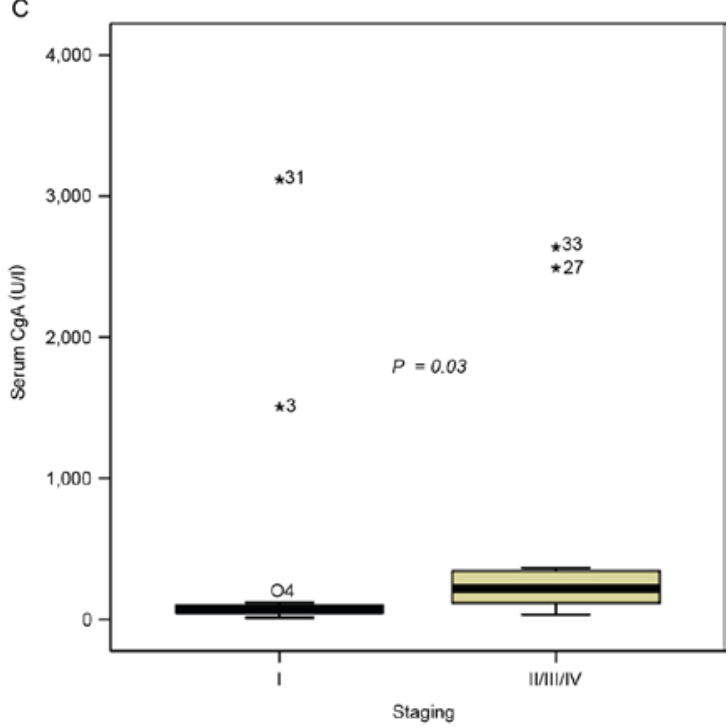

Figure 1. Plasma $\mathrm{CgA}$ levels according to tumor characteristics in pancreatic neuroendocrine tumors. Plasma CgA is significantly associated with (A) size, (B) metastasis and (C) staging. Asterisks denote outliers, with case numbers in brackets. $\mathrm{CgA}$, chromogranin A.
Table II. Sensitivity of $\mathrm{CgA}$ under different cutoff values.

\begin{tabular}{|c|c|c|c|}
\hline \multirow{2}{*}{$\begin{array}{l}\text { CgA cutoff } \\
\text { level, } U / 1\end{array}$} & \multicolumn{2}{|c|}{ Tumor location } & \multirow{2}{*}{$\begin{array}{c}\text { Overall } \\
\text { sensitivity }^{\mathrm{a}}\end{array}$} \\
\hline & Localized $^{\text {a }}$ & Metastasis $^{\mathrm{a}}$ & \\
\hline 94 & $29.6(8 / 27)$ & $87.5(7 / 8)$ & $42.9(15 / 35)$ \\
\hline 74 & $40.7(11 / 27)$ & $87.5(7 / 8)$ & $51.4(18 / 35)$ \\
\hline 64.3 & $51.9(14 / 27)$ & $87.5(7 / 8)$ & $60.0(21 / 35)$ \\
\hline
\end{tabular}



\section{Discussion}

The present study demonstrated that plasma $\mathrm{CgA}$ exhibited limited diagnostic value in early PNETs. Smaller and localized PNETs tended to exhibit lower plasma CgA levels. Although plasma $\mathrm{CgA}$ change was correlated with treatment response, this result was limited to the patients with high baseline $\mathrm{CgA}$ levels $(\geq 94 \mathrm{U} / \mathrm{l})$.

$\mathrm{CgA}$ is an acidic glycoprotein stored in the dense granules of NETs and co-released with peptide hormones (6). CgA is considered the most accurate tumor marker in the diagnosis of gastro-entero-pancreatic NETs (GEP-NETs), in comparison with other tumor markers, including urinary 5-hydroxyindoleacetic acid, neuron-specific enolase and carcinoembryonic antigen (7). The sensitivity and specificity of $\mathrm{CgA}$ differs and depends on numerous factors, such as the type of assay used, the cutoff value, tumor burden and organs involved (20). A small number of studies concerning the diagnostic value of plasma $\mathrm{CgA}$ in Asian populations are summarized in Table IV (11-14). The sensitivities and specificities of plasma $\mathrm{CgA}$ were 53.6-86.0 and 78.6-91.9\% respectively. However, these studies only enrolled a small number of early-stage PNETs. In the present study, the overall sensitivity was only $42.9 \%$, and the sensitivity of localized PNETs was even lower (29.6\%). The low sensitivity of plasma CgA identified in the patients was probably due to the small size of the tumors and the early stage. Among the localized tumors, the majority were $>2 \mathrm{~cm}(59 \%)$. A previous study indicated that small tumors may be associated with normal CgA levels (8). In concordance with previous studies, the present study demonstrated that plasma CgA levels were significantly associated with tumor size. Smaller tumors tended to exhibit lower plasma $\mathrm{CgA}$ levels. Conversely, the sensitivity of plasma $\mathrm{CgA}$ for patients with metastatic cancer was $87.5 \%$, which was comparable with a previous study conducted in Asia (11). CgA levels and their associated sensitivities depended on metastasis (21). Previous studies suggested that plasma $\mathrm{CgA}$ level was correlated with tumor mass and disease extent. $\mathrm{CgA}$ levels were higher in patients with extensive liver metastases compared with localized disease $(22,23)$. The present study also indicated that those patients with metastatic tumors tended to exhibit higher plasma $\mathrm{CgA}$ levels. The sensitivity of plasma $\mathrm{CgA}$ for patients with metastatic PNETs was relatively good in comparison to patients with localized PNETs.

Distant metastasis predicts a higher risk of mortality in PNETs $(24,25)$. Identification of reliable predictors of 
Table III. Univariate and multivariate analyses of predictors of metastasis.

\begin{tabular}{llll}
\hline Variables & HR $(95 \% \mathrm{CI})$ & Prvalue $(95 \% \mathrm{CI})$
\end{tabular}

Sex

Male vs. female

$0.93(0.19-4.50)$

$0.93(0.19-4.50)$

$3.33(0.65-17.18)$

$0.53(0.09-3.18)$

$2.05(1.13-3.74)$

$0.04(0.004-0.40)$

$16.63(1.75-158.09)$

Plasma $\mathrm{CgA}, \mathrm{U} / 1$

$\geq 94$ vs. $<94$

Head/neck vs. body/tail

Size, $\mathrm{cm}$

$>2$ vs. $\leq 2$

WHO grade

G1 vs. G2/3
HR, hazard ratio; CI, confidence interval; CgA, chromogranin A; WHO, World Health Organization.
0.927

0.927

0.150

0.490

0.019

$1.72(0.66-4.50)$

0.266

0.006

$0.09(0.57-202.8)$

0.112

0.014

$18.31(1.073-312.56)$

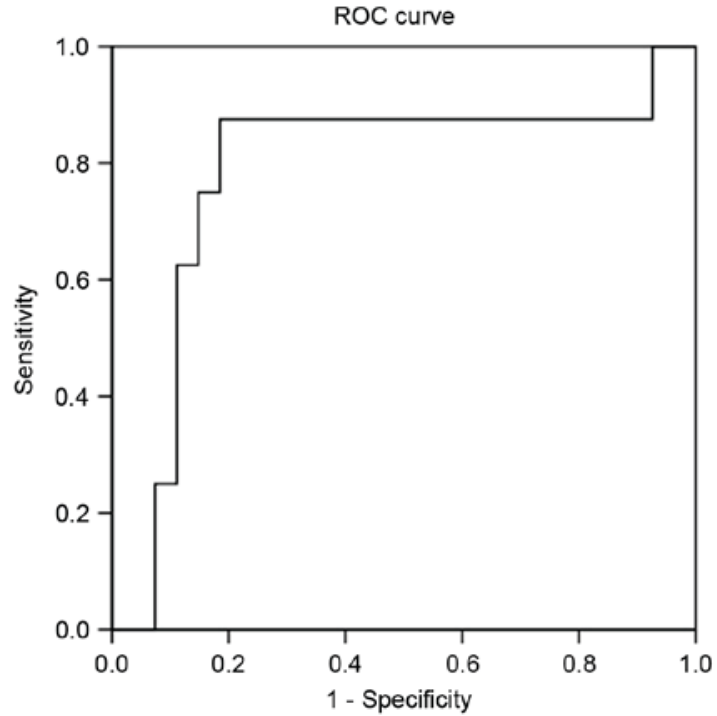

Figure 2. ROC curve of $\mathrm{CgA}$ obtained from 8 patients with metastasis and 27 patients without metastases. ROC, receiver operating characteristic.

metastasis has been the aim of numerous studies. Tumor size was the most commonly documented clinical predictor of metastasis (26). The risk of metastasis increased significantly if the tumor size exceeded $15 \mathrm{~mm}$. A small number of studies evaluated the association between the plasma CgA level and the risk of metastasis. Paik et al demonstrated that a high level of $\mathrm{CgA}(>156.5 \mathrm{U} / \mathrm{l})$ predicted distant metastasis in those patients with PNETs (27). In concordance with this study, the present study also demonstrated that plasma $\mathrm{CgA}$ was a reliable indicator of metastasis. When the cutoff value was $94 \mathrm{U} / 1$, the sensitivity and specificity were 87.5 and $70.4 \%$, respectively. Using $126 \mathrm{U} / 1$ as the best cutoff value, the sensitivity and specificity were 87.5 and $81.5 \%$, respectively. Identification

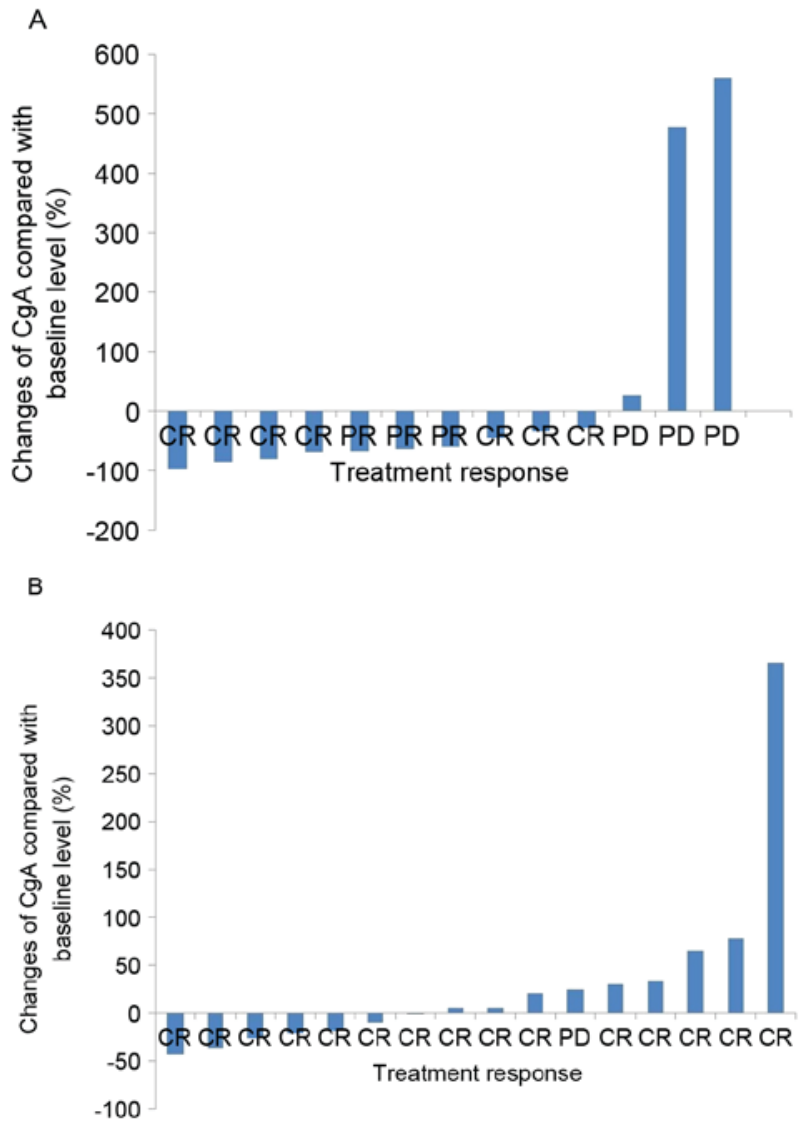

Figure 3. Correlation between plasma $\mathrm{CgA}$ change and treatment response among (A) high baseline group $(\mathrm{r}=0.523, \mathrm{P}=0.025)$ and $(\mathrm{B})$ low baseline group ( $\mathrm{r}=0.118, \mathrm{P}=0.587)$. $\mathrm{CR}$, complete response; $\mathrm{PD}$, progressive disease; CgA, chromogranin A.

of any metastatic tumors should thus be performed carefully once the PNET patient has demonstrated high CgA levels. 
Table IV. Summary of previous studies on the diagnostic value of CgA in Asian populations.

\begin{tabular}{lllcccr}
\hline \multirow{2}{*}{ First author } & \multicolumn{1}{c}{ Study design } & Assay type & Cutoff & Sensitivity, & Specificity, & vall \\
Han et al & Non-functional PNET $(\mathrm{n}=51)$ & Chromoa assay, ELISA & 64.3 & 63.2 & 91.2 & $(12)$ \\
Qiao et al & Non-insulinoma PNET $(\mathrm{n}=32)$ & Chromoa assay, ELISA & 74.0 & 65.6 & 91.9 & $(14)$ \\
Chou et al & GEP-NET $(\mathrm{n}=44)$ & Chromoa assay, ELISA & 94.0 & 86.0 & 88.0 & $(11)$ \\
Hijioka et al & PNET $(\mathrm{n}=69)$ & Chromoa assay, ELISA & 78.7 & 53.6 & 78.6 & $(13)$ \\
\hline
\end{tabular}

PNET, pancreatic neuroendocrine tumor; GEP, gastro-entero-pancreatic; CgA, chromogranin A.

Korse et al (28), demonstrated that a higher sensitivity of plasma $\mathrm{CgA}$ was noted in patients with well-differentiated tumors. The sensitivity was $\sim 68 \%$ for G1 NETs and $74 \%$ for G2 NETs. A much lower sensitivity (37\%) was noted in the patients with poorly differentiated NETs, which was probably due to the relative lack of large dense-core granules. The present study did not indicate a significant association between grade and plasma $\mathrm{CgA}$ level. This may be as only 2 cases of G3 NETs were enrolled in the series. The single patient with metastatic PNET and lower plasma $\mathrm{CgA}$ level exhibited grade G3 NET. Although plasma CgA was revealed to be useful in the detection of metastatic NETs (11), it may not diagnose those patients with poorly differentiated NETs.

Plasma CgA has been shown to be valuable in evaluating the treatment response of different therapies $(2,29)$. For patients with hepatic metastases from functional carcinoid tumors, Jensen et al (29), demonstrated that a reduction in $\mathrm{CgA}$ of $\geq 80 \%$ following cytoreductive surgery was predictive of the stabilization of disease. For patients with advanced PNETs, Yao et al (2) revealed that those patients with an early $\mathrm{CgA}$ response following treatment of everolimus experienced longer progression-free survival. Similar to previous studies, the present study also indicated that changes in plasma CgA were correlated with treatment response in those patients with high baseline $\mathrm{CgA}$ levels. However, this was not true for those patients with low baseline $\mathrm{CgA}$ levels, which implied that the role of $\mathrm{CgA}$ in evaluating treatment efficacy may be limited to patients with high baseline $\mathrm{CgA}$ levels.

Functional PNETs may present with various hormone syndromes, so they are usually detected at an earlier stage. By contrast, non-functional PNETs usually present with symptoms following local mass effects or metastatic disease at more advanced stages (5). In the present study, the majority of PNETs were non-functional and detected incidentally (60\%), with a localized (77\%) status. This is likely due to the widespread use of abdominal ultrasound in Taiwan. Routine ultrasound screening for patients with chronic hepatitis or abnormal liver function tests is extremely popular. PNETs localized in the body or tail may be easily detected by screening ultrasounds at an earlier stage.

A previous study indicated that high CgA levels (150-fold higher compared with the normal upper limit) in GEP-NETs were associated with MEN-1 (6). However, low diagnostic accuracy of plasma $\mathrm{CgA}$ in the detection of PNETs in patients with MEN-1 has been demonstrated by two other studies $(30,31)$. The plasma CgA test cannot replace the other established diagnostic tools in screening for early PNETs among patients with MEN-1. The results of the present study also indicated a limited use of plasma $\mathrm{CgA}$ in patients with MEN-1, although only 3 patients with MEN-1 were included and only 1 of these exhibited a higher CgA level.

There are a number of non-neoplastic causes of $\mathrm{CgA}$ elevation, including renal insufficiency, chronic hepatitis and drug use. Certain adenocarcinomas may also account for $\mathrm{CgA}$ elevation $(6,7)$. In the present study, the patients with liver failure, renal failure and other types of cancer were excluded. Although proton pump inhibitor use has been suggested to be a common cause for CgA elevation (32), only 2 cases in the present study were administered proton pump inhibitors and they exhibited different CgA responses (63.0 and 16,465.7 U/1). Additional interpretation with subgroup analysis was not feasible due to a relatively small number of patients with PNET in the present study. As PNET is an uncommon disease, future studies with multi-center cooperation may provide a more comprehensive view.

To conclude, the present study demonstrated that plasma CgA levels were associated with tumor size, metastasis status and tumor stage in diagnosing patients with PNET. Changes in plasma $\mathrm{CgA}$ levels were correlated with treatment response only in those patients with high baseline CgA levels. For early-stage PNETs, CgA exhibited a limited role in the diagnosis and evaluation of treatment response.

\section{Acknowledgements}

Not applicable.

\section{Funding}

Pinancial support was provided by Novartis (Taiwan) Co., Ltd., (Taipei, Taiwan) for the CgA test.

\section{Availability of data and materials}

All the datasets generated and analyzed in the present study are included in this published article. 


\section{Authors' contributions}

CMT designed the study, reviewed the article (Tables. 2 and 4) and drafted the manuscript. TYC drafted and revised the manuscript, collected data (FNA and tissue staining) and performed statistic analysis (Tables. 1 and 3). T-BC performed statistical analysis and revised the figures and tables (Tables. 1 and 3). YWT contributed to data collection (surgical tissue) and provided technical support. C-CC contributed to data collection (plasma chromogranin A) and was involved in the interpretation of data. JTL revised the study protocol and revised the manuscript. H-PW designed the study, contributed to data collection (patient's background) and performed critical revisions of the manuscript.

\section{Ethics approval and consent to participate}

The study protocol was approved by the Institutional Review Board of National Taiwan University Hospital (Taipei, Taiwan). Written informed consent for participation in the study was obtained from all participants.

\section{Consent for publication}

All study participants provided consent for the data to be published.

\section{Competing interests}

The authors declare that they have no competing interests.

\section{References}

1. Dasari A, Shen C, Halperin D, Zhao B, Zhou S, Xu Y, Shih T and Yao JC: Trends in the incidence, prevalence, and survival outcomes in patients with neuroendocrine tumors in the United States. JAMA Oncol 3: 1335-1342, 2017.

2. Yao JC, Pavel M, Phan AT, Kulke MH, Hoosen S, St Peter J, Cherfi A and Öberg KE: Chromogranin A and neuron-specific enolase as prognostic markers in patients with advanced pNET treated with everolimus. J Clin Endocrinol Metab 96: 3741-3749, 2011.

3. Milan SA and Yeo CJ: Neuroendocrine tumors of the pancreas. Curr Opin Oncol 24: 46-55, 2012.

4. Tsai HJ, Wu CC, Tsai CR, Lin SF, Chen LT and Chang JS: The epidemiology of neuroendocrine tumors in taiwan: A nation-wide cancer registry-based study. PLoS One 8: e62487, 2013.

5. Burns WR and Edil BH: Neuroendocrine pancreatic tumors: Guidelines for management and update. Curr Treat Options Oncol 13: 24-34, 2012.

6. Modlin IM, Gustafsson BI, Moss SF, Pavel M, Tsolakis AV and Kidd M: Chromogranin A-biological function and clinical utility in neuro endocrine tumor disease. Ann Surg Oncol 17: 2427-2443, 2010.

7. Lawrence B, Gustafsson BI, Kidd M, Pavel M, Svejda B and Modlin IM: The clinical relevance of chromogranin A as a biomarker for gastroenteropancreatic neuroendocrine tumors. Endocrinol Metab Clin North Am 40: 111-134, viii, 2011.

8. Vinik AI, Woltering EA, Warner RR, Caplin M, O'Dorisio TM, Wiseman GA, Coppola D and Go VL; North American Neuroendocrine Tumor Society (NANETS): NANETS consensus guidelines for the diagnosis of neuroendocrine tumor. Pancreas 39: 713-734, 2010.

9. Jilesen AP, Busch OR, van Gulik TM, Gouma DJ and Nieveen van Dijkum EJ: Standard pre- and postoperative determination of chromogranin a in resectable non-functioning pancreatic neuroendocrine tumors-diagnostic accuracy: NF-pNET and low tumor burden. Dig Surg 31: 407-414, 2014.
10. Shanahan MA, Salem A, Fisher A, Cho CS, Leverson G, Winslow ER and Weber SM: Chromogranin A predicts survival for resected pancreatic neuroendocrine tumors. J Surg Res 201: 38-43, 2016.

11. Chou WC,Hung YS, Hsu JT, Chen JS, Lu CH,Hwang TL, Rau KM, Yeh KY, Chen TC and Sun CF: Chromogranin A is a reliable biomarker for gastroenteropancreatic neuroendocrine tumors in an Asian population of patients. Neuroendocrinology 95: 344-350, 2012

12. Han X, Zhang C, Tang M, Xu X, Liu L, Ji Y, Pan B and Lou W: The value of serum chromogranin $\mathrm{A}$ as a predictor of tumor burden, therapeutic response, and nomogram-based survival in well-moderate nonfunctional pancreatic neuroendocrine tumors with liver metastases. Eur J Gastroenterol Hepatol 27: 527-535, 2015.

13. Hijioka M, Ito T, Igarashi H, Fujimori N, Lee L, Nakamura T, Jensen RT and Takayanagi R: Serum chromogranin A is a useful marker for Japanese patients with pancreatic neuroendocrine tumors. Cancer Sci 105: 1464-1471, 2014.

14. Qiao XW, Qiu L, Chen YJ, Meng CT, Sun Z, Bai CM, Zhao DC, Zhang TP, Zhao YP, Song YL, et al: Chromogranin A is a reliable serum diagnostic biomarker for pancreatic neuroendocrine tumors but not for insulinomas. BMC Endocr Disord 14: 64, 2014.

15. Yung RC, Otell S, Illei P, Clark DP, Feller-Kopman D, Yarmus L, Askin F, Gabrielson E and Li QK: Improvement of cellularity on cell block preparations using the so-called tissue coagulum clot method during endobronchial ultrasound-guided transbronchial fine-needle aspiration. Cancer Cytopathol 120: 185-195, 2012.

16. Chatzipantelis P, Salla C, Konstantinou P, Karoumpalis I, Sakellariou S and Doumani I: Endoscopic ultrasound-guided fine-needle aspiration cytology of pancreatic neuroendocrine tumors: A study of 48 cases. Cancer 114: 255-262, 2008.

17. Bosman FT, Carneiro F, Hruban RH and Theise ND: WHO classification of tumours of the digestive system: World Health Organization, 2010.

18. Edge SB, Byrd DR, Compton CC, Fritz AG, Greene F and Trotti A: AJCC Cancer Staging Manual. New York, NY: Springer, 2010.

19. Eisenhauer EA, Therasse P, Bogaerts J, Schwartz LH, Sargent D, Ford R, Dancey J, Arbuck S, Gwyther S, Mooney M, et al: New response evaluation criteria in solid tumours: Revised RECIST guideline (version 1.1). Eur J Cancer 45: 228-247, 2009.

20. Vinik AI, Silva MP, Woltering EA, Go VL, Warner R and Caplin M: Biochemical testing for neuroendocrine tumors. Pancreas 38: 876-889, 2009.

21. Nölting S, Kuttner A, Lauseker M, Vogeser M, Haug A, Herrmann KA, Hoffmann JN, Spitzweg C, Göke B and Auernhammer CJ: Chromogranin a as serum marker for gastroenteropancreatic neuroendocrine tumors: A single center experience and literature review. Cancers (Basel) 4: 141-155, 2012.

22. Campana D, Nori F, Piscitelli L, Morselli-Labate AM, Pezzilli R, Corinaldesi R and Tomassetti P: Chromogranin A: Is it a useful marker of neuroendocrine tumors? J Clin Oncol 25: 1967-1973, 2007.

23. Walter T, Chardon L, Chopin-laly X, Raverot V, Caffin AG, Chayvialle JA, Scoazec JY and Lombard-Bohas C: Is the combination of chromogranin A and pancreatic polypeptide serum determinations of interest in the diagnosis and follow-up of gastro-entero-pancreatic neuroendocrine tumours? Eur J Cancer 48: 1766-1773, 2012.

24. Cherenfant J, Stocker SJ, Gage MK, Du H, Thurow TA, Odeleye M, Schimpke SW, Kaul KL, Hall CR, Lamzabi I, et al: Predicting aggressive behavior in nonfunctioning pancreatic neuroendocrine tumors. Surgery 154: 785-793, 2013.

25. Metz DC and Jensen RT: Gastrointestinal neuroendocrine tumors: Pancreatic endocrine tumors. Gastroenterology 135: 1469-1492, 2008

26. Kishi Y, Shimada K, Nara S, Esaki M, Hiraoka N and Kosuge T: Basing treatment strategy for non-functional pancreatic neuroendocrine tumors on tumor size. Ann Surg Oncol 21: 2882-2888, 2014.

27. Paik WH, Ryu JK, Song BJ, Kim J, Park JK, Kim YT and Yoon YB: Clinical usefulness of plasma chromogranin a in pancreatic neuroendocrine neoplasm. J Korean Med Sci 28: 750-754, 2013.

28. Korse CM, Taal BG, Vincent A, van Velthuysen ML, Baas P, Buning-Kager JC, Linders TC and Bonfrer JM: Choice of tumour markers in patients with neuroendocrine tumours is dependent on the histological grade. A marker study of Chromogranin A, Neuron specific enolase, Progastrin-releasing peptide and cytokeratin fragments. Eur J Cancer 48: 662-671, 2012. 
29. Jensen EH, Kvols L, McLoughlin JM, Lewis JM, Alvarado MD, Yeatman T, Malafa M and Shibata D: Biomarkers predict outcomes following cytoreductive surgery for hepatic metastases from functional carcinoid tumors. Ann Surg Oncol 14: 780-785, 2007.

30. de Laat JM, Pieterman CR, Weijmans M, Hermus AR, Dekkers OM, de Herder WW, van der Horst-Schrivers AN, Drent ML, Bisschop PH, Havekes B, et al: Low accuracy of tumor markers for diagnosing pancreatic neuroendocrine tumors in multiple endocrine neoplasia type 1 patients. J Clin Endocrinol Metab 98: 4143-4151, 2013.

31. Granberg D, Stridsberg M, Seensalu R, Eriksson B, Lundqvist G, Oberg K and Skogseid B: Plasma chromogranin A in patients with multiple endocrine neoplasia type 1. J Clin Endocrinol Metab 84: 2712-2717, 1999.
32. Giusti M, Sidoti M, Augeri C, Rabitti C and Minuto F: Effect of short-term treatment with low dosages of the proton-pump inhibitor omeprazole on serum chromogranin A levels in man. Eur J Endocrinol 150: 299-303, 2004. Attribution-NonCommercial-NoDerivatives 4.0 International (CC BY-NC-ND 4.0) License. 